\title{
Singularities that induce a breakdown of determinism in mechanics
}

\author{
Mike R. Jeffrey \\ Department of Engineering Mathematics, University of Bristol, Bristol BS8 1TR, UK
}

February 25, 2013

\begin{abstract}
We will show that determinism can break down at certain singularities that arise in the mechanics of semi-rigid bodies. An example system consists of a wheel slipping and rolling along a surface, such that dry friction applies a torque to the wheel via a standard nonlinear tyre-like interaction. In a certain configuration the force on the wheel and its ensuing motion acquire many possible values. The subsequent motion is therefore infinitely sensitive to perturbation, constituting a point of non-determinism in an otherwise deterministic system. Moreover the device returns to the singularity repeatedly, so that it suffers repeated bursts of unpredictability, constituting an extreme non-deterministic form of chaos. A non-deterministic chaotic attractor was found for a range of parameters and tyre/friction models in (2013). Here, we explore the building blocks of this dynamics by stripping the model down to its key elements. We investigate the non-deterministic phenomenon when subjected to relaxations of the model that allow for smoothing, noise, and hysteresis. This is part of a wider theory currently emerging in the study of dynamical systems that undergo sharp switches in behaviour, whereby singularities can violate traditional rules of smooth dynamical systems.
\end{abstract}

\section{INTRODUCTION}

In dynamical systems modeling, discontinuities are widely used to model abrupt switches such as electronic relays, mechanical impact, and stick-slip motion; see e.g. $(1999,2008)$. Their application is increasingly reaching beyond traditional problems to include extreme nonlinearity and slow-fast timescale separation in electronics, mechanics, and the life sciences; e.g. (2011, 2010, 2003). Previous studies have progressed largely by extending established concepts from dynamical systems theory for smooth models, into a theory of piecewise-smooth dynamics. In recent years, however, certain discontinuity-induced phenomena have come to light that have no counterpart in smooth systems. They represent novel topological sources of unpredictable behaviour, and offer insight into the idealizations that are made in deriving empirical models of switching behaviour.

In (2010, 2011, 2013), certain physical models were shown to exhibit singularities at which determinism breaks down, meaning that, when the system is in one particular configuration, solutions of its differential equations become multi-valued.
The singularity occurs due to switching associated with stick-slip mechanics in (2013), and with a superconductivity threshold in (2011). A general classification of such singularities appears in (2011). Perhaps the most provocative outcome of these studies is an unconventional form of chaotic dynamics, driven by a loss of determinism at the so-called Teixeira singularity (2011). The singularity can occur in MIMO (multiple-input multiple-output) circuit models (2010), and was shown to create non-deterministic chaos in a mechanical friction model (2013).

The simplicity of the phenomenon itself is somewhat obscured in these preliminary studies, due to irrelevant nonlinearities in the practical models, and due to the rigours of normal form analysis in the general theory. Is the loss of determinism an artefact of modelling fundamentally smooth underlying physics using discontinuities, or does it provide insight into behaviour that is encountered daily, but dismissed as unmodelled error? To begin addressing these questions we focus here on the simple mechanism behind non-deterministic chaos, and the complex behaviour it creates; both appear for the first time in this paper. 
In the mathematical sense, the configurations of interest are described by so-called two-fold singularities. They are generic in systems with at least two dimensions with one switch (2009), or two dimensions with more than one switch (2013). In the relatively young field of piecewise-smooth systems, however, it remains unclear whether 'genericity' implies a prevalence of such configurations in real systems. It also remains unclear exactly how one should recognize such behaviour in experimental data. Here we will present simulations of the typical dynamics in a stripped down model, which we then subject to smoothing, hysteresis, and noise.

We introduce the phenomenon of non-deterministic chaos by deriving a caricature encapsulating only the essential features of previous mechanical and electronic models. Our starting point is a mechanical system involving friction between a tyre and a dry revolving surface. In this conceptual model introduced in (2013), a rolling wheel experiences a torque due to standard tyre-like contact forces, and this creates highly unpredictable rattling due to a singularity. The wheel system is introduced in sec.2, and in sec.3 the concept of non-deterministic chaos is introduced. A caricature model is derived in sec.4, generalized to consider smoothing, noise, and hysteresis, and then these systems are simulated in sec.5.

\section{A MECHANICAL EXAMPLE}

The mechanical device depicted in fig. 1 consists of a disc rotating with constant angular speed $\omega_{0}$, on top of which sits a wheel, which is free to roll along a line placed a distance $d$ from the disc axis. The wheel is fixed at an angle $\gamma$ to this line. A tyre model for the contact force between the wheel and the disc then translates the frictional contact force into a torque on the wheel. The specifics of the tyre model (discussed in (2013)) are not vital to the dynamics observed.

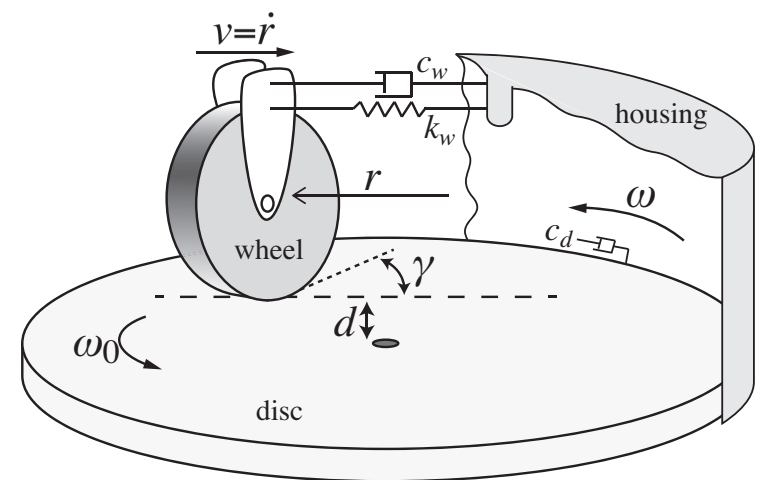

Figure 1: Sketch of the wheel assembly. Friction between the revolving disc, and a tyre on the wheel rim, apply linear and angular forces on the wheel as it rolls along the dashed line. The wheel-disc contact can stick or slip at different times.
The constraint by which the wheel moves along a line is also not crucial here, but is achieved by mounting it on a slider, embedded in a housing which is essentially another disc mounted coaxially above the lower disc; constants $c_{d}, c_{w}$, and $k_{w}$, give damping and spring coefficients between the housing and lower disc (subscript $h$ ) or wheel (subscript $w$ ).

For the time-varying displacement $r$ and velocity $v$ of the wheel, and the angular speed of the housing, $\omega$, one obtains dynamical equations of the form

$$
\begin{aligned}
\dot{r} & =v \\
\dot{v} & =a_{1}(r, v, \omega)-b_{1}(r, v, \omega) \operatorname{sign}(h) \\
\dot{\omega} & =a_{2}(r, v, \omega)-b_{2}(r, v, \omega) \operatorname{sign}(h)
\end{aligned}
$$

where $a_{i}$ and $b_{i}$ are nonlinear functions. The $a_{i}$ capture the system geometry, while the $b_{i}$ contain the contact model (for details we refer the reader to (2013)). More crucial is the function $\operatorname{sign}(h)$, which expresses the relative direction of the wheel's motion with respect to the disc, measured in the plane of the wheel. The function $h$ is

$h=\left(v-d\left(\omega-\omega_{0}\right)\right) \sin \gamma+r\left(\omega-\omega_{0}\right) \cos \gamma$.

When this quantity vanishes for a period of time it means the wheel and the disc are in sticking contact. The equations governing sticking motion can be derived using Filippov's method (1988, 2003), which we describe briefly in the next section, and the resulting equations can be solved to simulate the device's dynamics (see (2013)). An alternative to Filippov's method, particularly suited to numerical simulation, is to smooth out the discontinuity in the sign function at $h=0$, so that it transitions smoothly between \pm 1 in a small region $|h|<\varepsilon$ for small $\varepsilon$. The system is simulated by this method in fig. 2 .

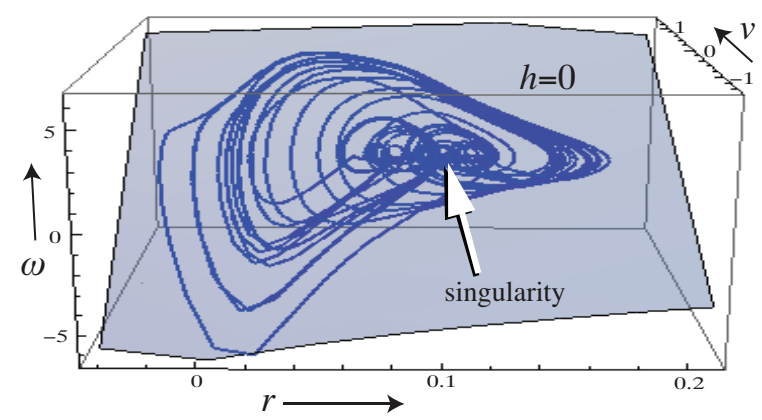

Figure 2: Simulation of the dynamics of the wheel assembly in fig. 1, indicating the sticking surface $h=0$, and the singularity. A solution of (1) is found by replacing the function $\operatorname{sign}(h)$ by $\tanh (h / \varepsilon)$, with stiffness parameter $\varepsilon=10^{-3}$; [all simulations made in Mathematica ${ }^{\mathrm{TM}}$ using NDSolve].

Although a smoothed-out model of the system is solvable using standard numerical tools, the system is then more stiff, as controlled by the parameter $\varepsilon$. Be- 
sides affecting computation time, smaller $\varepsilon$ introduces instability to the simulation in ways that are poorly understood. As we describe below, the dynamics in fig. 2 involves the funnelling of solutions into and out of a singularity. In the discontinuous system this means that all trajectories at some time pass through the same point. That point represents a configuration of the physical device which can be reached from any initial conditions, and from which many different configurations of the system are possible at some later time. If the discontinuity is smoothed out, the system becomes extremely sensitive to initial conditions near the singularity. These problems are general properties, not specific to one system, but attributable to a specific class of singularities. Let us therefore isolate the features of the model that create these conditions.

\section{A SCHEMATIC DEFINITION}

At the heart of the mechanical system in fig. 2 lies a Teixeira singularity, a generic feature of discontinuous systems first studied in $(1988,1990)$. The discontinuity surface can be divided into regions where trajectories cross through it, are attracted to it, or are repelled from it. All three types of behaviour are brought together at the singularity, as shown in fig. 3 .

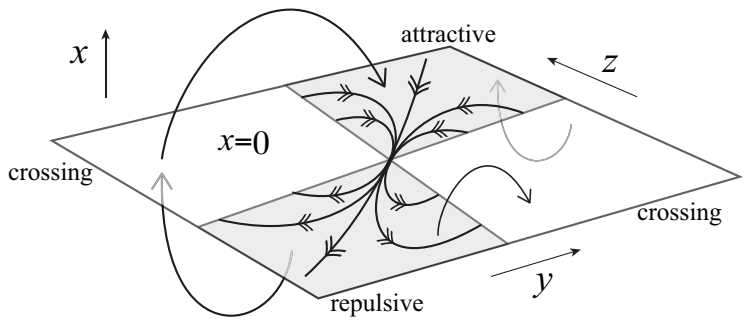

Figure 3: The Teixeira singularity - an isolated point in three dimensions, or an $n-3$ dimensional set in $n$ dimensions. At a discontinuity surface $(x=0)$ trajectories can cross (unshaded regions) or stick (double arrows, shaded regions). The sticking regions attract or repel the surrounding trajectories. The boundaries of the different regions intersect at the singularity.

Consider a system

$(\dot{x}, \dot{y}, \dot{z})=\left\{\begin{array}{lll}\mathbf{f}^{+}(x, y, z) & \text { if } \quad x>0 \\ \mathbf{f}^{-}(x, y, z) & \text { if } \quad x<0\end{array}\right.$

(we can increase the dimensionality, but three is the minimum required to understand the singularity), where $\mathbf{f}^{ \pm}$are smooth vector fields. The system switches between $\mathbf{f}^{+}$and $\mathbf{f}^{-}$at the discontinuity surface $x=0$ (which was $h=0$ in sec.2). The standard way to define dynamics at $x=0$ (1988) is to take a linear combination of the two vector fields, say $\mathbf{g}^{\lambda}=\lambda \mathbf{f}^{+}+(1-\lambda) \mathbf{f}^{-}$, and if there exists $\lambda \in[0,1]$ for which $\mathbf{g}^{\lambda}$ lies tangent to the surface, then $\mathbf{g}^{\lambda}$ defines a sliding vector field on $x=0$. This 'sliding' dynamics prescribes 'sticking' motion (double arrows in fig. 3), and the requirement $\lambda \in[0,1]$ (meaning $\mathbf{g}^{\lambda}$ lies somewhere between $\mathbf{f}^{+}$and $\mathbf{f}^{-}$), confines sticking to the shaded regions in fig. 3, where $\mathbf{f}^{+}$and $\mathbf{f}^{-}$have opposing directions with respect to $x=0$. Elsewhere, the fields $\mathbf{f}^{ \pm}$cause trajectories to cross $x=0$ transversally. The boundaries between sticking and crossing are where $\mathbf{f}^{+}$or $\mathbf{f}^{-}$lie in the plane $x=0$, and a singularity forms where both $\mathbf{f}^{+}$and $\mathbf{f}^{-}$lie in the plane.

Various forms for this singularity are possible. Here we are interested in the case shown in fig. 3, when all sticking trajectories traverse the singularity, but moreover, they do so in the counterintuitive direction from the attractive region to the repulsive region. A local normal form for this scenario is known $(1988,1990$, 2009), given by (3) with

$\mathbf{f}_{0}^{+}(y)=(-y, 1, v), \quad \mathbf{f}_{0}^{-}(z)=(z, w, 1)$

in terms of constants $v, w<0$ satisfying $v w>1$; from these equations one can easily sketch fig. 3 . The point $(x, y, z)=(0,0,0)$ is the Teixeira singularity. Observe that a whole volume of trajectories will fall into the attractive sticking region, and then enter the singularity. Conversely, a whole volume of trajectories can be traced backwards in time to the repulsive sticking region, and thence to the singularity.

It is easy to show that passage through the singularity takes a finite amount of time (2009). This implies that when a trajectory traverses the singularity, its possible histories and possible futures are momentarily multi-valued - an instant of non-determinism.

The dynamics becomes more striking if we introduce a feedback to the system, as exists in the mechanical problem of sec.2, so that every trajectory, once ejected from the repulsive side of the singularity, is eventually re-injected via the attractive side (see fig. 4). Although the system's immediate future becomes unpredictable when it arrives at the singularity, whichever exit path it follows will lead eventually back to the singularity, giving rise to repeating yet unpredictable dynamics, describable as chaotic (2009).

Without specifying the method of re-injection, we can understand very powerfully what this nondeterministic chaos represents. Taking any two points in the chaotic set, we can find a finite-time trajectory that connects them (see fig. 4). This means any configuration of the system can evolve into any other configuration (within the chaotic set). In a deterministic system, chaos arises when all trajectories pass exponentially close to one other given sufficient time. In a discontinuous system this is taken to an extreme, so that all trajectories intersect given sufficient time, when they pass through the singularity. 


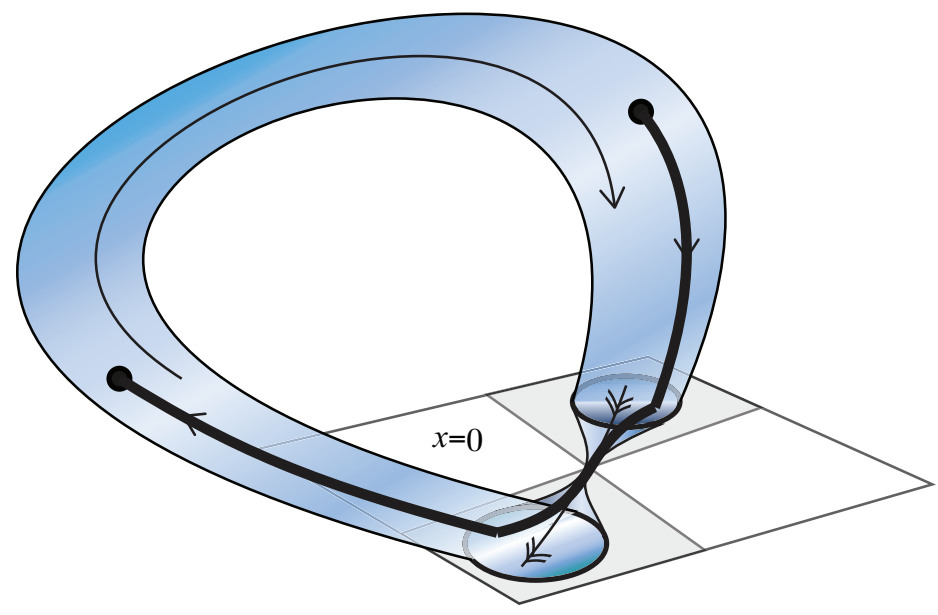

Figure 4: The mechanism of non-deterministic chaos: a Teixeira singularity on $x=0$, and a re-injection, that forces all trajectories to repeatedly visit the singularity. Any two points can be connected by a trajectory passing through the singularity, so which of these connections will persist under perturbation?

If we approximate the system by something deterministic, which of the possible trajectories through the singularity will persist? In the spirit of deterministic chaos, we expect the singularity to lead to an infinite sensitivity to initial conditions. To investigate its effect on the dynamics let us now give a specific form for the re-injection described above. To the local normal form (3), we must add a nonlinear term that dominates far from the singularity, steering trajectories from the repulsive sticking region, back towards the attractive region.

\section{ANALYSIS OF A SIMPLIFIED MODEL}

The required effect is easily achieved by adding a drift term in the $(0,1,1)$ direction, of strength $\alpha$, and an attraction term $(0,-y,-z)$ of strength $\beta$, for some constants $\alpha, \beta>0$. Multiplying these by $|\mathbf{x}|^{2}=x^{2}+y^{2}+$ $z^{2}$ ensures they only become significant far from the singularity, and we obtain a model

$$
\begin{aligned}
& \mathbf{f}^{+}(x, y, z)=(-y, 1, v)+(0, \alpha-\beta y, \alpha-\beta z)|\mathbf{x}|^{2} \\
& \mathbf{f}^{-}(x, y, z)=(z, w, 1)+(0, \alpha-\beta y, \alpha-\beta z)|\mathbf{x}|^{2} .
\end{aligned}
$$

Any initial condition in a neighbourhood of the origin leads to a trajectory of the form shown in fig. 5 .

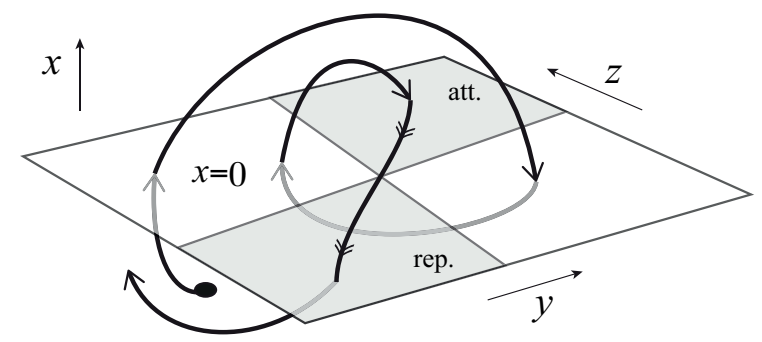

Figure 5: A typical trajectory of (5).
By simulating this system, respecting crossing and sticking dynamics as described in the previous section, one finds that there exists a region around the origin where all trajectories intersect the origin in forward and backward time.

At the origin, the singularity dictates that no single path can be chosen uniquely from the continuum of possible trajectories. Nor is there any unique way to resolve this ambiguity. The discontinuous model is representative of a range of systems that become extremely sensitive to initial conditions near the singularity. To gain some insight we will smooth out the discontinuity, which restores determinism, and then add noise and hysteresis to the model. We define these models in the rest of this section, then we simulate each of them in sec.5.

As a preparatory step, we relax the definition of (3) so that the switch takes place over a region $|x|<\varepsilon$, which collapses to a discontinuity at $x=0$ as $\varepsilon$ tends to zero. We replace the system (3) with

$$
(\dot{x}, \dot{y}, \dot{z})=\mathbf{f}^{\epsilon}(x, y, z) \text {, }
$$

for some small $\varepsilon \geq 0$, such that

$\lim _{\varepsilon \rightarrow 0} \mathbf{f}^{\varepsilon}(x, y, z)=\left\{\begin{array}{lll}\mathbf{f}^{+}(y) & \text { if } \quad x>0, \\ \mathbf{f}^{-}(z) & \text { if } \quad x<0 .\end{array}\right.$

This can be achieved by letting $\mathbf{f}^{\varepsilon}$ be a linear combination of $\mathbf{f}^{ \pm}$, and considering

$$
(\dot{x}, \dot{y}, \dot{z})=\mathbf{f}^{\epsilon}=\mathbf{f}^{-}+\left(\mathbf{f}^{+}-\mathbf{f}^{-}\right) \phi
$$

where (7) places a requirement on $\phi$ that

$\phi=\{1$ for $x>+\varepsilon \& 0$ for $x<-\varepsilon\}+O(\varepsilon)$.

\subsection{Smoothing}

A smooth system is now found by letting $\phi$ be a smooth function $\phi=\phi(x / \varepsilon)$ (e.g. for the simulations to follow, we will mainly consider $\phi=\frac{1}{2}[1+$ $\tanh (x / \varepsilon)])$. Substituting $x=\varepsilon u$ gives

$(\varepsilon \dot{u}, \dot{y}, \dot{z})=\mathbf{f}^{-}(\varepsilon u, y, z)+\left[\mathbf{f}^{+}(\varepsilon u, y, z)-\mathbf{f}^{-}(\varepsilon u, y, z)\right] \phi(u)$.

The result is a system where $u$ varies on a fast timescale $t / \varepsilon$, while $y$ and $z$ vary on the slow timescale $t$. For such slow-fast systems, much is known about the correspondence between, for example, Fenichel's slow manifolds (1979), which occupy a region of space where the $u$ component of $\mathbf{f}^{-}+\left(\mathbf{f}^{+}-\mathbf{f}^{-}\right) \phi$ vanishes, and the sticking regions which form as $\varepsilon \rightarrow 0$ (2007); these are recent but well established developments, so we do not explore them more deeply here. 


\subsection{Noise}

A thorough study of how noise distributions propagate in the system is beyond the scope of the present paper. For brevity, we simulate noise in the system (8) by adding a random perturbation of size $\varepsilon$ to the state $(x(t), y(t), z(t))$, after each time interval $\Delta t=0.3$. We let $\phi=\frac{1}{2}[1+\tanh (x / \varepsilon)]$.

\subsection{Hysteresis}

To introduce hysteresis to (8), let $\phi$ be a variable (which adds a dimension to the system), by adding to (8) the equation

$\varepsilon^{\prime} \dot{\phi}=\psi\left([x+\varepsilon(2 \phi-1)] / \varepsilon^{\prime}\right)-\phi$

where $\psi$ is another function that satisfies

$$
\psi=\left\{1 \text { for } w>+\varepsilon^{\prime} \& 0 \text { for } w<-\varepsilon^{\prime}\right\}+O(\varepsilon)^{\prime}
$$

for some small $\varepsilon^{\prime}$. Then $\phi$ is attracted (on the fast timescale $\left.t / \varepsilon^{\prime}\right)$ towards +1 if $x+\varepsilon(2 \phi-1)>\varepsilon^{\prime}$ and towards 0 if $x+\varepsilon(2 \phi-1)<-\varepsilon^{\prime}$. For the simulations to follow we let $\psi=\frac{1}{2}\left[1+\tanh \left(w / \varepsilon^{\prime}\right)\right]$ and $\varepsilon^{\prime}=10 \varepsilon$.

One further generalization that will not be considered here (due to space restrictions, and because the effect is in some ways similar to hysteresis), is to consider a delay in the switching function $\phi$, for example $\mathbf{f}^{\varepsilon}=\mathbf{f}^{-}+\left(\mathbf{f}^{+}-\mathbf{f}^{-}\right) \phi(x(t-\delta) / \varepsilon)$ for some $\delta>0$.

\section{SIMULATIONS}

The purpose of the simulations below is to demonstrate the sensitivity of the model (5). For a standardized method we solve the ordinary differential equations using the default NDSolve function in Mathematica $^{\mathrm{TM}}$. All simulations start from an initial point $(x, y, z)=(0.2,0,-1)$, with $\mathbf{f}^{ \pm}$fixed by parameters $v^{+}=-1.2, v^{-}=-1.4, \alpha=10^{-3}, \beta=10^{-5}$, and with stiffness parameter $\varepsilon=10^{-3}$.

Each image in fig. 6 shows a single trajectory, winding around and revisiting the singularity repeatedly over a time $t=150$. Despite some overall similarity, the detailed shape of the orbits differs markedly, exhibiting less organisation in the basic smoothed model (i) than when hysteresis is applied in (ii), and revealing changes in structure sensitive to random noise in (iii)-(iv).

Given the complexity of the phase space trajectories, more insight is gained by taking a Poincaré section, recording only coordinates where a trajectory passes through the plane $y=0$. All of the sections below are simulated over a time $t=2 \times 10^{5}$, but plotting only the latter half of the trajectory to omit transients.
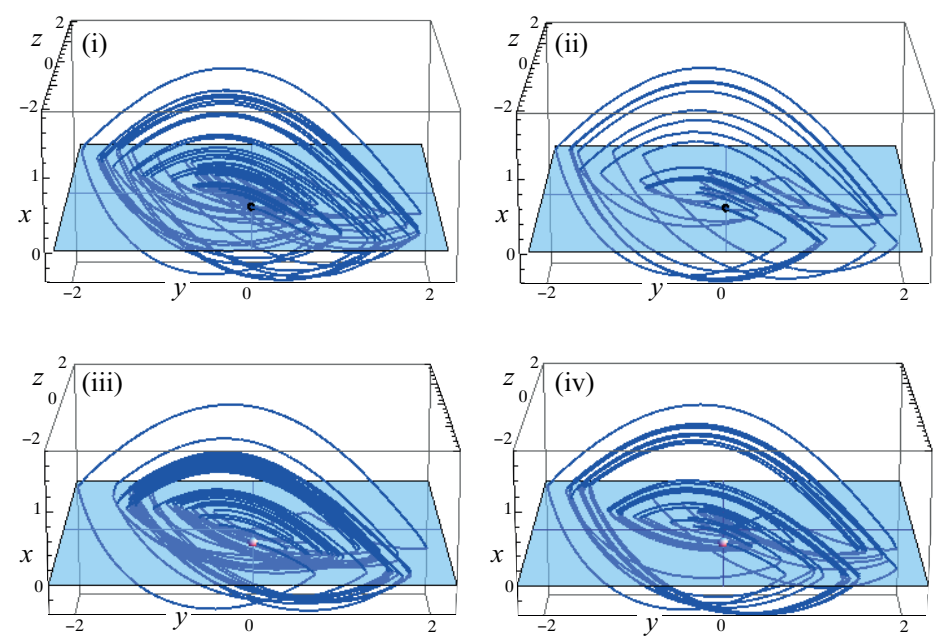

Figure 6: Simulation of: (i) the system in sec.4.1 for $\phi=\frac{1}{2}[1+$ $\tanh (x / \varepsilon)]$; (ii) the hysteretic system (10), (iii-iv) the noisy system described in sec.4.2, showing two separate simulations from the same initial conditions.

We first highlight the sensitivity of the system to the method of simulation itself. One could use different numerical iteration schemes with different levels of precision to solve the dynamical system. We show one simple variant in fig. 7 , dividing the total trajectory time into fixed intervals that force the adaptive NDSolve function to restart periodically. With this seemingly redundant change, the numerical simulation settles to different attractors (for the same time and initial conditions); we present examples of a periodic orbit, a period three orbit, and the seeming appearance of deterministic chaos.
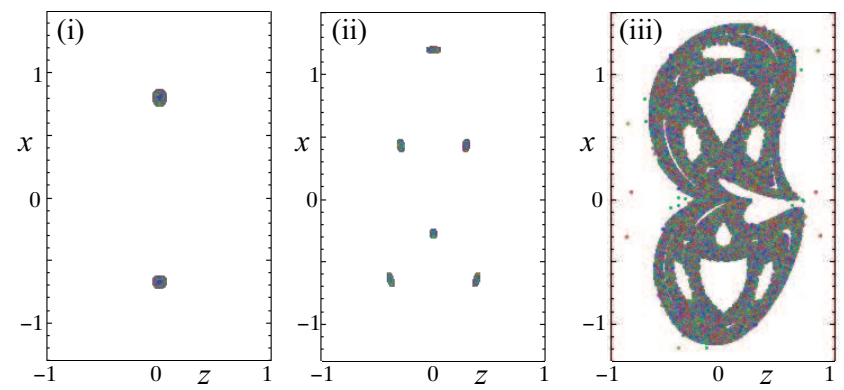

Figure 7: Poincaré sections for the system in sec.4.1 with $\phi=$ $\frac{1}{2}[1+\tanh (x / \varepsilon)]$, for different iteration schemes: dividing the trajectory into time intervals (i) 20 , (ii) 40, (iii) 100 .

One assumes that a more sophisticated simulation will reveal which result from fig. 7 is correct, but the ambiguity is compounded by other sensitivities in the system, such as the choice of how to smooth out the discontinuity. In fig. 8 we repeat the simulation in fig. 7(iii) using a perturbed smoothing function $\phi$, which still tends to $\operatorname{sign}(x)$ as $\varepsilon \rightarrow 0$; this is no less valid, therefore, as an approximation of the discontinuous system (5). Small changes in the size of this perturbation give different attractors, as before. 

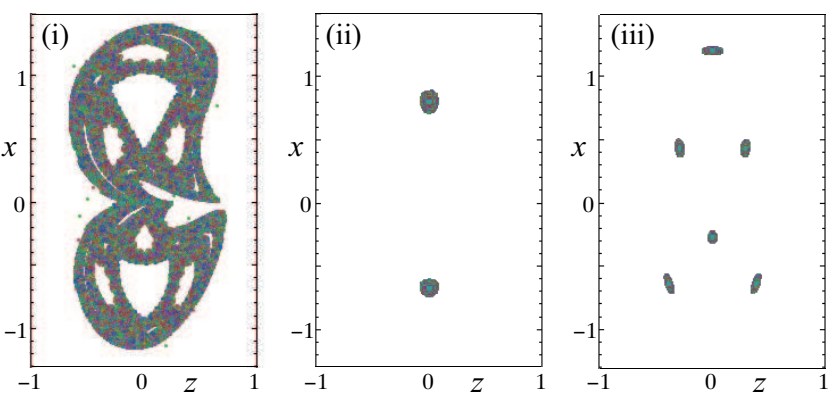

Figure 8: Poincaré sections for the system in sec.4.1 with $\phi=$ $\frac{1}{2}[1+\tanh (x / \varepsilon)]+\sigma \sqrt{\varepsilon} e^{-x^{2}}$ and: (i) $\sigma=0.01$, (ii) $\sigma=0.1$, (iii) $\sigma=0.13$.

Finally, in fig. 9, more physically realistic scenarios are simulated by repeating fig. 7(iii) with hysteresis added in (i) or noise added in (ii-iii).
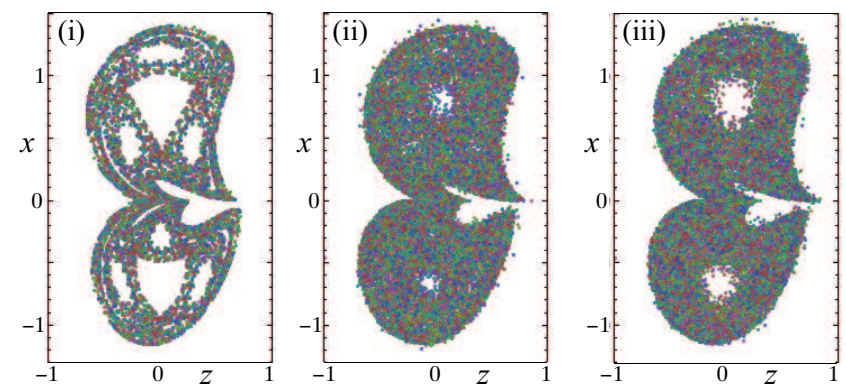

Figure 9: Poincaré sections for the system in sec.4.1 with $\phi=$ $\frac{1}{2}[1+\tanh (x / \varepsilon)]:$ (i) with hysteresis as described in sec.4.3; (ii) with noise as described in sec. 4.2 for two different runs.

These various simulations show for the first time the sensitive behaviour associated with nondeterministic chaos. We observe that many different deterministic systems lie close to the discontinuous system. The ambiguity of selecting between outcomes can be attributed to the existence of a nondeterministic singularity in the discontinuous limit, which amplifies even tiny changes in parameters. For some parameters the deterministic system exhibits stable periodic orbits (figs. 7(i-ii) and 8(ii-iii)), while for others the dynamics appears chaotic over some intricate attractor (figs. 7(iii), 8(i), 9(iii)), only some of which is washed out by noise (figs. 9(i-ii)). The important point is that all of these systems differ by only small changes in the defining model. Under these small changes the system jumps between regimes of periodicity or (deterministic) chaos.

\section{CLOSING REMARKS}

Renewed interest in recent years in a general theory of switching systems relevant to engineering applications, has inspired closer inspection of certain singularities where switching causes extreme sensitivity in a model, modeled dynamically by a breakdown of determinism. In this paper we described a mechanical model that exhibits such behaviour, constructed a toy model to study the phenomenon itself, and simulated its dynamics under relaxations of the model that take account of smoothing, hysteresis, and noise. This revealed unpredictability, partially organised by underlying structure, that survives from such a singularity when the idealization of discontinuity is relaxed.

\section{REFERENCES}

Bachar, G., E. Segev, O. Shtempluck, E. Buks, \& S. W. Shaw (2010). Noise induced intermittency in a superconducting microwave resonator. EPL 89(1), 17003.

Brogliato, B. (1999). Nonsmooth mechanics - models, dynamics and control. Springer-Verlag (New York).

Colombo, A., M. di Bernardo, E. Fossas, \& M. R. Jeffrey (2010). Teixeira singularities in 3D switched feedback control systems. Syst.\&Contr.Letters 59(10), 615-622.

Colombo, A. \& M. R. Jeffrey (2011). Non-deterministic chaos, and the two-fold singularity in piecewise smooth flows. SIAM J. App. Dyn. Sys. 10, 423-451.

Desroches, M. \& M. R. Jeffrey (2011). Canards and curvature: nonsmooth approximation by pinching. Nonlinearity 24, 1655-1682.

di Bernardo, M., C. J. Budd, A. R. Champneys, \& P. Kowalczyk (2008). Piecewise-Smooth Dynamical Systems: Theory and Applications. Springer.

Fenichel, N. (1979). Geometric singular perturbation theory. J. Differ. Equ. 31, 53-98.

Filippov, A. F. (1988). Differential Equations with Discontinuous Righthand Sides. Kluwer Academic Publ.

Jeffrey, M. R. (2011). Non-determinism in the limit of nonsmooth dynamics. PRL 106(254103), 1-4.

Jeffrey, M. R. (2013). On the problem of uniqueness in multiple switching dynamics: hierarchy \& isonomy. in draft.

Jeffrey, M. R., A. R. Champneys, M. di Bernardo, \& S. W. Shaw (2010). Catastrophic sliding bifurcations and onset of oscillations in a superconducting resonator. Phys. Rev. E 81(1), 016213-22.

Jeffrey, M. R. \& A. Colombo (2009). The two-fold singularity of discontinuous vector fields. SIAM Journal on Applied Dynamical Systems 8(2), 624-640.

Jeffrey, M. R. \& S. J. Hogan (2011). The geometry of generic sliding bifurcations. SIREV 53(3), 505-525.

Jeffrey, M. R. \& R. Szalai (2013). Non-deterministic dynamics of a mechanical systems. in draft.

Kuznetsov, Y. A., S. Rinaldi, \& A. Gragnani (2003). One-parameter bifurcations in planar Filippov systems. Int.J.Bif.Chaos 13, 2157-2188.

Teixeira, M. A. (1990). Stability conditions for discontinuous vector fields. J. Differ. Equ. 88, 15-29.

Teixeira, M. A., J. Llibre, \& P. R. da Silva (2007). Regularization of discontinuous vector fields on $R^{3}$ via singular perturbation. J.Dyn.Diff.Equat. 19(2), 309-331. 